\title{
A Conceptual Framework for a Computer-Assisted, Morphometric-Based Phytolith Analysis and Classification System
}

\author{
Rand R. Evett ${ }^{\mathrm{a}}$ and Rob Q. Cuthrell ${ }^{\mathrm{b}}$ \\ aUniversity of California, Berkeley \\ Dept. of Environmental Science, Policy, and Management \\ 130 Mulford Hall \\ Berkeley, CA 94720-3114 \\ ${ }^{b}$ University of California, Berkeley \\ Archaeological Research Facility \\ 2251 College Building \\ Berkeley, CA 94720-1076 \\ Corresponding Author: \\ Rand R. Evett \\ revett@sonic.net \\ University of California, Berkeley \\ Dept. of Environmental Science, Policy, and Management \\ 130 Mulford Hall \\ Berkeley, CA 94720-3114 \\ 707-538-7869
}

\begin{abstract}
Although automated approaches to shape analysis and object classification have been widely applied in the biological sciences, technical and time considerations have limited their use in phytolith research. As advanced microscopy systems become more affordable and accessible and digital imaging software provides a wider range of sophisticated analytical tools, there is increased potential for effective use of machinevision and automation in phytolith research. In this paper, we describe technical limitations of phytolith imaging and identify several techniques that might improve results. Drawing on examples of software developed for related disciplines, we then describe a conceptual framework for development and integration of automated phytolith analysis software for: separating phytoliths from non-phytolith material in digital images; segmentation of phytolith boundaries; quantitative phytolith feature extraction, including a discussion of potentially more powerful, non-traditional parameters of phytolith shape and texture; phytolith classification and identification; and phytolith database image retrieval. While recognizing the difficulty of implementing this framework and the need for extensive empirical testing of suggested approaches on phytoliths, we examine the possibility of aggregating quantitative phytolith data collected in studies worldwide to construct a cloud-based database of phytolith images with associated morphotype data.
\end{abstract}

\section{Keywords}


silica phytoliths; emerging techniques; multivariate analysis; automated classification; staining; morphometrics; elliptic Fourier analysis

\section{Highlights (3-5 bullet points, up to 85 chars each)}

- New techniques from other biological disciplines can be used to analyze and classify phytoliths

- Applying sophisticated morphometric measures may greatly improve phytolith classification

- An integrative, semi-automated software package is a long-term goal for phytolith research

\section{Introduction}

For most of its history as a science, phytolith analysis has relied on visual identification and classification of phytolith morphotypes based on qualitative criteria. The advent of microcomputers in the 1980s spurred interest in phytolith morphometrics, a quantitative approach to phytolith analysis based on precisely measured size and shape parameters. While several pioneering studies have demonstrated the potential efficacy of quantitative approaches (Albert et al. 2009; Ball et al. 1993, 1996, 1999, 2006; Berlin et al. 2003; Lu et al. 2009; Pearsall et al. 1995; Russ and Rovner 1989), technical issues make these processes difficult and time-consuming, limiting their use by phytolith researchers.

Recently, advanced microscopy systems have become more affordable and accessible to researchers, and the availability of user-friendly digital imaging software now permits implementation of a wider range of more sophisticated analytical tools. Computer-assisted analysis, already increasingly applied in related disciplines within the biological sciences, can potentially have an increased role in phytolith research, enabling the development of semi-automated quantitative classification systems and the construction of accessible morphometric databases.

Although phytolith researchers have successfully addressed many research questions through qualitative approaches to phytolith analysis, quantitative approaches, if practicable, offer several significant advantages (Ball et al. 2015; Lootens et al. 2007):

1) Observer bias is minimized - phytolith measurement and classification do not depend on the degree of training of the practitioner, a substantial source of potential error (Green et al. 2014; Mander et al. 2014).

2) There is more consistency between labs - data gathered by researchers worldwide will be more compatible for integration.

3) Parameters that more effectively differentiate similar morphotypes can be identified and disseminated. 
4) Data are on a continuous scale, reflecting the complexity of phytolith morphology in nature. From these data, morphotypes can be precisely and quantitatively defined through multivariate analysis after data collection.

5) Automatic data processing is possible. With development of automated systems, samples may be analyzed more efficiently than is possible with manual techniques, leading to more productive, less costly studies.

Drawing inspiration from approaches developed in other biological disciplines, we describe a conceptual framework for the development of quantitative, computerassisted methods for phytolith analysis.

We present a sequence of research topics that could be pursued to construct an efficient, widely applicable, integrative, and accessible system for morphometric phytolith analysis over the coming decades. These topics include: data acquisition; data analysis and classification; integration of data and analytical tools; and data dissemination and accessibility (Figure 1).

\section{Image Data Acquisition}

A primary challenge that must be addressed is developing microscopic methods that will permit accurate and efficient digital representation of phytolith shape, either in two or three dimensions.

\subsection{Two dimensional phytolith imaging: Edge detection and shape feature extraction}

\subsubsection{Staining phytoliths for enhanced imaging}

Phytoliths are clear particles of silica, often several microns thick. Defining the boundary of a phytolith under a light microscope is problematic because only a thin slice of a phytolith is in sharp focus at one time, although an extended depth of focus approach may improve boundary detection (Wu and Wang 2009). Boundary definition is also difficult because there is low contrast due to minimal difference between the specific gravity of phytoliths and the mounting medium, as well as a strong halo effect (Kloster et al. 2014). For these reasons, many phytolith morphometric studies to date have relied on the expensive, time-consuming procedure of preparing scanning electron microscope images of phytoliths (Ball et al. 1993, 1996, 1999, 2006).

The accuracy of boundary detection in transmitted light microscopy may be considerably improved by staining phytoliths. Phytoliths in plant tissues have been successfully stained using phenol crystals, methyl red, crystal violet lactone, and silver amine chromate (Benvenuto et al. 2013; Dayanandan et al. 1983; Johansen 1940; Kao et al 2008; Kaufman et al. 1985). The latter three stains are adsorbed to the silica surface by bonding with silanol groups (Kaufman et al. 1985). However, each of these stains has limitations for use in imaging modern reference material. Phenol stain results in light rose coloration, unlikely to aid in boundary detection (Benvenuto et al. 2013). Although methyl red, crystal violet lactone, and silver amine chromate can produce saturated colors in phytoliths that aid in imaging, these stains are not consistently adsorbed by modern reference phytoliths (Blecher et al. 2012; Kao et al. 2008). 
Kaufman et al. (1985) suggested that due to the similarity in molecular composition between phytoliths and commercial silica gel, dyes that stain silica gel may also be useful for staining phytoliths. Researchers assessing the use of silica gel or other silica products for removal of dye from wastewater have reported effective adsorbance of crystal violet, methylene blue, and malachite green, with greater adsorption generally associated with higher pH levels (Fisk et al. 2005; Kannan et al. 2008; Zhao et al. 2008). Methylene blue and crystal violet can be used to stain the majority of phytoliths in modern reference samples, as well as non-phytolith aggregates of amorphous silica present in phytolith samples recovered from soils (Cuthrell 2013). However, these stains are only adsorbed by a minority of soil phytoliths, and generally only in damaged portions of the phytolith (Cuthrell 2013; Evett and Cuthrell 2013). These stains have been used in a semi-automated digital imaging process to effectively differentiate phytoliths from non-phytolith silica in soil samples (ibid.). However, to date no effective methods to stain soil phytoliths for transmitted light microscopy have been reported. Methods developed to stain the frustules of mature and fossil diatoms could be tried on phytoliths derived from soils (Friedrichs 2013; Friedrichs et al. 2012; Kucki et al. 2006). Further research based on techniques that alter surface chemistry to attach fluorescent dye to phytoliths and diatoms may eventually enable staining of soil phytoliths with visible dyes (Descles et al. 2008; Fishkis et al. 2010; Soukup et al. 2014).

\subsubsection{Image segmentation and automated object measurement}

Current protocols for phytolith morphometrics require either direct measurement of distance parameters with an eyepiece micrometer or a ruler on a phytolith image, or manually digitizing phytolith boundaries on an image to enable more sophisticated quantitative shape analysis using macros in image processing software such as ImageJ (Ball et al. 2015; Out et al. 2014). In order to make rapid phytolith measurement practical for routine use, methods must be developed that will allow software to automatically define, or segment, object boundaries on slide images containing many phytolith and non-phytolith particles.

Automatic image segmentation has been successfully used in a number of scientific and medical fields and has recently been applied to microfossils (Du Buf and Bayer 2002; Kloster et al. 2014; Han et al. 2014; Lagerstrom et al. 2015). Particularly promising is SHERPA, a program developed for digitally processing images of diatom frustules (Kloster et al. 2014), which are also composed of silica and similar to phytoliths regarding problems with segmentation. SHERPA automatically tries several segmentation approaches before producing an optimal digital boundary estimate, an approach that may be tested on phytoliths.

Semi-automated object segmentation of phytoliths and non-phytoliths on slides has been successfully applied to distinguish stained non-phytolith silica from unstained phytoliths using Olympus Microsuite, GIMP, ImageJ, and CombineZP software (Cuthrell 2013; Evett and Cuthrell 2013). Object boundaries were enhanced by producing a composite image from a Z-stack (a vertical sequence of images) processed in CombineZP, edges were enhanced through a contrast filter, and a simple threshold was applied manually to detect object boundaries (Figure 2). Although the boundaries of most phytoliths were represented accurately, due to low contrast between edges and 
mounting media, some phytoliths were inaccurately imaged as "hollow" objects. If effective staining techniques can be developed, staining phytoliths prior to imaging would mitigate this problem.

Phytoliths are extremely diverse objects, with large differences in size, shape, thickness, color, surface ornamentation, and degree of silicification. Flat, thin morphotypes, such as grass long cells, are amenable to accurate automatic twodimensional segmentation if all of the silica is completely stained. Grass short cell morphotypes are not as planar in shape, and the shape resulting from two-dimensional segmentation is thus more dependent on phytolith orientation. The degree of difficulty and amount of testing required for adequate two-dimensional segmentation of problematic morphotypes, such as spheroids and appendages, should not be underestimated.

After a phytolith boundary has been segmented, the next step required to build a semi-automated phytolith analysis procedure is to transfer the data into an automated shape feature extraction program. The current cutting edge in phytolith research are macros in ImageJ or other digital image analysis software that process manually or automatically defined boundary data and output geometric size and shape data (Ball et al. 2015; Cuthrell 2013; Out et al. 2014). SHERPA has progressed considerably further than this with diatom analysis, allowing researchers to automatically input boundary data from the segmentation process into a module that manipulates the data to produce a much wider range of shape descriptors than ImageJ; this module could potentially be applied to phytolith analysis after extensive testing on a range of morphotypes.

\subsection{Three dimensional phytolith imaging}

In confocal laser scanning microscopy (CLSM), light interference from the surrounding regions of an object are eliminated by sequentially imaging a series of points to build a coplanar raster image representing a section through the object, typically using phase contrast or fluorescent imaging (Webb 1996). This permits much more accurate representation of two dimensional sections of relatively thick transparent objects than is possible using conventional transmitted light microscopy. A series of parallel two dimensional sections through an object can be combined to produce a three dimensional model of the object.

Effective, simple methods for fluorescent labeling of phytoliths will need to be developed before CLSM can be used for phytolith analysis. In a study of phytolith transport in soils, Fishkis et al. (2010) successfully stained modern phytoliths with fluorescein isothiocyanate. Soukup et al. (2014) used phytolith autofluorescence in a basic aqueous solution to image phytoliths using conventional fluorescence microscopy, but autofluorescence may not produce sufficient intensity for effective confocal imaging. Several studies of diatoms used fluorescent stains incorporated into diatoms in vivo, which may be useful for studying formation of phytoliths in plant tissues (Descles et al. 2008; Groger et al. 2008; Kucki et al. 2006).

Although no published studies have applied confocal microscopy to phytoliths, two diatom studies illustrate the potential utility of CLSM for three-dimensional phytolith analysis. Friedrichs (2013) used a relatively simple procedure to stain fossil diatoms ranging in age from the Pleistocene to Paleocene epochs and produced high fidelity 
three-dimensional models. Friedrichs et al. (2012) combined SEM photogrammetry with CLSM to create higher resolution three-dimensional models of modern diatoms. If these techniques can be successfully adapted to meet the unique requirements of phytolith analysis, three-dimensional phytolith models can improve analysis in several major ways. First, manipulation of models in software alleviates the difficult problem in conventional transmitted light microscopy of attempting to record images of phytoliths at particular orientations. Second, three-dimensional models can be virtually sectioned at any location and orientation, facilitating comparison of object boundary shapes in two dimensions. Third, availability of three-dimensional phytolith models will allow phytolith analysts to take advantage of emergent techniques for statistical analysis of threedimensional shape as these develop, increasing the potential to distinguish subtle morphotype differences.

\section{Shape and Feature Data Acquisition}

After an image has been segmented, a variety of techniques may be used to describe phytolith shape and other features quantitatively.

\subsection{Conventional geometric shape parameters}

More than 25 years ago, Russ and Rovner (1989) used image analysis software on phytolith boundary data to obtain measures of a number of basic geometric size and shape parameters. Despite advances in research on powerful new methods to mathematically characterize shape, subsequent phytolith researchers have continued to utilize these parameters (Ball et al. 2015). A large number of mathematical shape descriptors and parameters have been developed for a variety of applications (Russ 2011; Yang et al. 2008). More sophisticated parameters may enable researchers to better discriminate between highly similar phytolith morphotypes.

The simple geometric parameters traditionally employed in phytolith morphometrics, including roundness, solidity, and compactness, use geometric formulas and dimensionless ratios based on various measurements of distance (Ball et al. 2015). Many geometric parameters that have been utilized elsewhere but not applied to phytolith morphometrics, including center of gravity and axis of inertia (Yang et al. 2008), as well as new parameters, such as alternative measures of geometric circularity (Krieger 2014), should be tested for efficacy on phytolith data.

While geometric parameters have been widely adopted because of their ease of calculation and are useful as filters to identify gross differences in shape, much potentially useful shape information is lost if these are the only factors used (Yang et al. 2008). Mathematically sophisticated boundary-based descriptors of shape, including fractal (Oleschko et al. 2004), harmonic, and topological approaches (Russ 2011), more completely summarize shape information.

\subsection{Elliptic Fourier analysis of shape}

One of the most promising approaches to shape analysis for phytolith researchers, widely applied in numerous studies in a variety of disciplines, is elliptic 
Fourier analysis (EFA; Kuhl and Giardina 1982; Iwata and Ukai 2002). This harmonic approach produces an infinite series of elliptic Fourier descriptors (EFDs) that retain all of an object's shape information, such that the object can be precisely reconstructed from these descriptors (Nixon and Aguado 2002). In EFA, EFD coefficients are often used as inputs for multivariate analysis, typically principal components analysis (PCA; e.g., Chitwood et al. 2013; Mebatsion 2012a) or linear discriminant analysis (LDA; e.g., Vieira et al. 2014; Williams et al. 2012). One advantage of EFA for phytolith research is that the EFDs can be mathematically normalized to be invariant to translation (location of an object in an image), rotation (translation around an axis), and scale (shape is independent of size). This characteristic of EFA is crucial for the study of phytolith morphotypes that do not have readily identifiable landmarks.

Because most of the useful shape information required to distinguish objects such as phytoliths is normally contained in 5-10 descriptors, resulting data sets can be large (four coefficients per descriptor generates ca. 20-40 variables). To reduce the number of variables, most researchers subject EFD data to principal components analysis, automatically performed by the computer program SHAPE (Iwata and Ukai 2002), prior to conducting further analysis. EFDs often key on subtle aspects of shape that may be poorly represented using geometric parameters or go unnoticed by researchers. In this way, EFA may enable differentiation of phytolith morphotypes that are very similar in appearance.

A survey of the literature reveals myriad applications of EFA to shape analysis and classification of diverse objects, including: sand grains (Suzuki et al. 2014); cereal grains (Mebatsion 2012a); plant leaves (Chitwood et al. 2013); plant roots (Lootens et al. 2007); pollen grains (Bonhomme et al. 2013); grape seeds (Milanesi et al. 2014; Orru et al 2013); coffee beans (Oyama 2014; Severa et al. 2012); coral sclerites (Carlo et al. 2011); arthropods (Dujardin et al. 2014); and galaxies (Dutta et al. 2013). EFA has also been successfully applied to classification of three-dimensional shapes (Godefroy et al. 2012). One promising recent development is the application of invariant elliptic Fourier descriptors (IEFDs; Nixon and Aguado 2002) to distinguish highly similar cereal grains (Mebatsion et al. 2012b); in this study, only five IEFD descriptors were required to successfully classify 15,000 specimens. The large body of literature supporting the successful use of EFA to describe and classify shapes suggests the approach should be extensively tested on phytoliths.

\subsection{Emergent shape descriptors}

Additional approaches to quantifying shape features are described in Yang et al. (2008). Based on the unique requirements for phytolith morphometrics, the most promising of these approaches include: Zernike moments (Athilakshm and Wahi 2014; Cao et al. 2013; Garcia-Ordas et al. 2014; Khotanzad and Hong 1990; Mahi and Kaouadji 2014; Tsolakidis et al. 2014); Chebyshev moments (Dai et al. 2009; Marcos and Cristobal. 2013; curvature scale-space (Kumar et al. 2012); bounding-box splitting (Bauckhage and Tsotsos 2005); wavelet analysis (Essendelft 2013; Gui et al. 2014); shock graphs (Macrini 2003; Sebastian et al. 2004; Siddiqi et al. 1999); and boundaryline analysis (Liang et al. 2012). Several of these approaches have been tested and compared with each other on non-phytolith data sets, including: artificial image 
databases (Selvarajah and Kodituwakku 2011); feathers (Sheets et al. 2006); wheat seeds (Williams et al. 2013); plant roots (Lootens et al. 2007); and pollen (Lagerstrom et al. 2015).

\subsection{Surface texture descriptors}

Identification of phytolith morphotypes that have surface ornamentation, which is currently defined qualitatively (ICPN 2005), may benefit from the quantitative description of texture. A number of mathematical measures of texture have been developed (Nixon and Aguado 2002) and applied to discrimination of objects, such as pollen, that rely on texture as a major distinguishing feature (Mander et al. 2013; Marcos and Cristobal 2013; Marcos et al. 2015; Pozo-Banos et al. 2015; Redondo et al. 2015).

Shape and texture descriptors should be tested on standardized phytolith image sets to compare their ability to discriminate phytolith morphotypes and incorporated into semi-automated phytolith analysis software packages when appropriate. The availability of many independent measures of shape for inclusion in classification models may improve a researcher's ability to reliably distinguish between highly similar phytolith morphotypes.

\section{Data Analysis and Phytolith Classification}

After size, shape, and texture parameters of phytoliths in a study are measured and extracted, data from known phytolith specimens can be analyzed using multivariate or other statistical analyses to determine quantitative diagnostic criteria for classification of unknown phytolith morphotypes. In several cases, linear discriminant analysis (LDA) has been successfully used to accomplish this task in phytolith research (Ball et al. 1999; Iriarte 2003; Russ and Rovner 1989; Zhang et al. 2011; Zhao et al. 1998). However, LDA is only one of a suite of multivariate statistical classification approaches that have been applied to phytoliths, including cluster analysis, principal components analysis, multidimensional scaling, and other methods (Chavez and Thompson 2006; Hart and Matson 2009; Hart et al. 2011).

During the last two decades, there has been intense interest in developing more sophisticated classification approaches that take advantage of rapid advances in computing power. Examples of these approaches that have been applied to other disciplines and may be suitable for phytolith analysis include: the support vector machine approach to distinguish plant leaves and fish otoliths (Reig-Bolano et al. 2011; Tsolakidis et al. 2014); neural networks for coffee beans and regions in satellite images (Mahi and Kaouadji 2014; Oyama 2014); decision trees for vegetation types (Tooke et al. 2009); and random forests for diatoms (Dimitrovski et al. 2011). Efficacy of some of these approaches has been demonstrated for pollen images by Lagerstrom et al. (2015).

\section{Data and Analytical Integration and Automation}

\subsection{Develop an efficient system for phytolith image retrieval}


After a large amount of phytolith data has been collected, analyzed, and classified, the next requirement for an automated system is to develop an effective search engine that will allow a researcher to compare shape and other feature parameters of an unknown phytolith specimen with documented specimens and to retrieve similar specimens for inspection. A number of approaches to content-based image retrieval (CBIR) have recently been developed (Datta et al. 2005), and researchers have used CBIR to automatically assign semantic tags to images. Combining CBIR and morphometric data, individual phytolith specimens could be automatically assigned relevant qualitative morphotype labels (semantic tags), such as "bilobate" or "globular." This would speed the process of phytolith labeling, improve the consistency of the assignment of descriptive labels, and allow researchers to search the database using traditional phytolith descriptive terms. This approach has already been applied successfully to an artificial shape database (Kassimi and El beqqali 2011) and to large pollen databases (Han et al. 2014; Koutsoukos 2013).

\subsection{Combine all modules into one user-friendly phytolith analysis computer program}

One long-term goal of quantitative phytolith analysis may be development of software that combines all of the processes described above into one user-friendly program. In this type of system, a researcher would input a micrograph image of a slide field containing multiple objects, including phytoliths and non-phytoliths. The computer program would quantitatively determine whether objects are likely to be phytoliths, accurately segment the boundaries of phytolith specimens, and calculate geometric, elliptic Fourier, or other size, shape and texture parameters. Using a classification algorithm, each unknown phytolith would then be identified by anatomical (e.g., celltype) and taxonomic origin. Accessing a database, the program would also retrieve a number of similar images for comparison and evaluation of classification results.

While such a program may seem ambitious, several biological object classification programs with many of these capabilities have already been developed. Most of the elements required for a semi-automated phytolith classification program already exist in some form in other disciplines, but much research and testing will be required to refine modules and combine them into an integrated software package designed to address the specific challenges of quantitative phytolith analysis.

For example, SHERPA is a diatom frustule segmentation and feature extraction program that automatically applies several segmentation procedures to each object on a slide (Kloster et al. 2014). Frustules are differentiated from non-diatom objects based on comparison of elliptic Fourier descriptors of outlines produced by each of the segmentation procedures with those from a shape template library. Identified objects are ranked according to quality scores that indicate how close their outlines match template shapes to distinguish objects that are clearly diatoms and to indicate objects which require further boundary definition. There are interactive procedures at each step that enable the user to manually examine and improve problematic contours. Considerable shape data are extracted from each object and can be exported into an external statistical program for further analysis and classification. SHERPA has been successfully used for diatom reference material but has not yet been applied to the more difficult task of identifying fossil diatoms in a paleoecological study. Addition of 
classification and image retrieval modules would make this a complete, semi-automated program.

Classfynder is an image processing and classification program developed for pollen on microscope slides (Holt et al. 2011; Lagerstrom et al. 2015). In this system, a robotic module automatically scans a slide, uses coarse shape analysis of each object to separate pollen grains from other objects, accurately segments objects identified as pollen by creating a high-resolution single composite image based on a nine-image Zstack of the pollen grain, calculates 43 size, shape, and texture feature parameters, and classifies each pollen grain using a neural network trained on a pollen reference library. However, the program does not have image retrieval capability. Another program for pollen, developed by Han et al. (2014), starts with manually selected images of pollen grains, uses multiple segmentation procedures to automatically produce the best boundary outline, and extracts 69 feature parameters. The program has no classification module, but does have a module that uses the feature parameters to automatically assign applicable semantic labels to each image, enabling image retrieval. Combining Classfynder with the Han et al. (2014) program and adding an image retrieval module could eventually lead to a complete, semi-automated pollen analysis system.

Among several programs that have been developed to automate plant leaf segmentation and classification, by far the most tested is Leafsnap, a mobile application installed on over one million devices that processes an input image of a single leaf to identify taxonomic origin (Kumar et al. 2012). The program segments the leaf using color parameters, extracts features using an integrated curvature measures algorithm, identifies the leaf using a nearest neighbor procedure that quantitatively compares the unknown specimen with a reference library, and retrieves several images from the library for the user to examine and make the final decision on leaf identity.

\section{Data Accessibility and Dissemination}

Another goal of quantitative phytolith analysis is to aggregate all phytolith morphometric data collected by researchers into an open international database, using standardized protocols for morphometric data collection, analysis and archiving to ensure data compatibility (e.g., Ball et al. 2015). The current trend in biological science is toward "big data", digitizing every study and object of interest and storing this information in the cloud (Marx 2013). There have been concerted efforts to digitize natural history collections (Gries and Soltis 2014), as well as proxy paleoecological data from a variety of sources (http://www.ncdc.noaa.gov/data-access/paleoclimatologydata), in order to take advantage of the power of big data. Phytcore, an online database consisting of phytolith images uploaded by researchers worldwide, has recently become operational (Albert et al. 2014). While Phytcore does not currently have the capability of storing morphometric data for each image, adopting a bio-image analysis and management platform such as Bisque (Kvilekval et al. 2010) would enable morphometric information in the form of metadata to be attached to each image. While assembling a comprehensive quantitative database of phytoliths found in important taxa worldwide is a considerable task that may require decades, developing standardized 
protocols for morphometric data collection, analysis and archiving that will ensure data compatibility (e.g., Ball et al. 2015) is an immediate priority.

\section{An Integrated, Semi-Automated Phytolith Analysis System}

We have presented a conceptual blueprint of the elements that will be required to achieve a semi-automated phytolith analysis system. Figure 3 illustrates the workflow of such a system. The phytolith analysis software package would be capable of exchanging data with the online international phytolith database. Users conducting analysis of unknown phytoliths would choose to input either two- or three-dimensional data.

Two dimensional data could be in the form of a single phytolith image or of an entire slide scanned on a motorized stage. After users specify the spatial resolution of the image (e.g., $\mu \mathrm{m} /$ pixel), the program would apply algorithms to segment image objects, creating polygons for shape analysis. The system would then apply a general classification algorithm to differentiate phytoliths from non-phytolith objects and from overlapping objects on the slide, presenting users with ambiguous objects for acceptance or rejection. Once a sample set of phytolith objects is selected, the program would apply algorithms, based on a combination of the analytical approaches described above, to classify phytoliths and output the most likely anatomical and taxonomic identifications, measures of identification certainty, and pictures of other examples of the phytolith type from the online database. The user would have the option to use a number of classification systems, based on the phytolith type or the geographical origin. The program would also return data and pictures of other phytolith types likely to be confused with the analyzed specimen for user evaluation. Users would have the option to upload data from their analysis directly to the international phytolith database.

In the near future, three-dimensional data would probably be in the form of a closed polygon model of a single phytolith. After the user defines the scale, the program would use 3D EFA or other techniques in a classification algorithm and export results in the same way as the two dimensional data.

An important aspect of this integrated phytolith analysis system is the ability to collect and analyze data from reference materials and to upload these data to the international phytolith database. Using either two- or three-dimensional data, users would specify the anatomical and taxonomic origin of selected phytoliths, as well as the shape analysis algorithms applied to each phytolith; results of the analysis would be integrated into existing data sets used to create the classification algorithms after upload. Photographs or 3D models of analyzed objects would be uploaded to the database as well. Through the analytical software, users would have the option to download reference phytolith data or archaeological and paleoecological data sets to develop and refine classification algorithms.

\section{Conclusion}

Following the trajectory of other disciplines that analyze biological shape data, we anticipate that phytolith analysis will become increasingly quantitative in the future, with greater emphasis on computer-assisted automation. Because related disciplines 
have progressed further along this path, phytolith researchers can draw on the considerable body of research available in the literature. However, a large amount of testing and additional methodological development specific to phytoliths, far too much for an individual researcher or research group, will be required to achieve an automated analysis program. There are significant hurdles to overcome. Developing any kind of automated system is a complex process where many considerations must be taken into account. For example, specific measurement parameters required will vary depending on the objectives of each study, and each parameter utilized must be extensively tested on calibration and application sets before it can be confidently applied automatically. Biases resulting from machine vision must be identified and quantified. For phytoliths in particular, all morphotypes utilized in a study must be tested because, given their enormous range in size, shape, and surface ornamentation, many morphotypes may have distinct measurement issues. It is possible that testing will show that some of the components of the conceptual automated system we have described are not yet practical for phytolith research without further technological developments, but we believe increasingly automated analysis is a valuable long-term goal. Researchers can contribute to progress toward automated analysis by talking to each other about what is needed, developing standardized protocols when appropriate, and testing, developing, reporting on, and sharing successful new approaches they have tried.

\section{Acknowledgements}

This research was supported by NSF grant \#CHE-1314073.

\section{References}

Albert, R.M., Bamford, M.K., Cabanes, D., 2009. Palaeoecological significance of palms at Olduvai Gorge, Tanzania, based on phytolith remains, Quaternary International 193, 41-48.

Albert, R.M., Esteve, X., Portillo, M., Rodriguez-Cintas, A., Cabanes, D., Esteban, I., Hernandez, F., 2014. PhytCore: Phytolith Reference Collection. Accessed March 22, 2015. Accessible online at http://www.gepeg.org/enter_PCORE.html.

Athilakshm, R., Wahi, A., 2014. Improving object classification using Zernike moment, radical Cheybyshev moment based on square transform features: A comparative study, World Applied Sciences Journal 32, 1226-1234.

Ball, T., Davis, A.L., Evett, R.R., Ladwig, J.L., Tromp, M., Out, W., Portillo, M., 2015. Morphometric analysis of phytoliths: Recommendations towards standardization from the International Committee for Phytolith Morphometrics, Journal of Archaeological Science (Submitted).

Ball, T.B., Gardner, J.S., Anderson, N., 1993. A typologic and morphometric study of variation in phytoliths from einkorn wheat (Triticum monococcum), Canadian Journal of Botany 71, 1182-1192. 
Ball, T.B., Gardner, J.S., Anderson, N., 1999. Identifying inflorescence phytoliths from selected species of wheat (Triticium monococcum, $\mathrm{T}$. dicoccon, $\mathrm{T}$. dicoccoides, and $\mathrm{T}$. aestivum) and barley (Hordeum vulgare and H. spontaneum) (Gramineae), American Journal of Botany 86, 1615-1623.

Ball, T.B., Gardner, J.S., Brotherson, J.D., 1996. Identifying phytoliths produced by the inflorescence bracts of three species of wheat (Triticum monococcum L., T. dicoccum Schrank and T. aestivum L.) using computer assisted image and statistical analyses, Journal of Archaeological Science 23, 619-632.

Ball, T.B., Vrydaghs, L., Van Den Hauwe, I., Manwaring, J., De Langhe, E., 2006. Differentiating banana phytoliths: wild and edible Musa acuminata and Musa balbisiana, Journal of Archaeological Science 33, 1228-1236.

Bauckhage, C., Tsotsos, J.K., 2006. Bounding box splitting for robust shape classification, 2005 International Conference for Image Processing II, 478-481.

Benvenuto, M.L., Honaine, M.F., Osterrieth, M.L., 2013. Amorphous silica biomineralizations in Polytrichum strictum Menzies ex Brid. (Bryophyta), Journal of Bryology 35, 112-118.

Berlin, A.M., Ball, T.B., Thompson, R., Herbert, S.C., 2003. Ptolemaic agriculture, "Syrian wheat," and Triticum aestivum, Journal of Archaeological Science 30, 115-121.

Blecher, I.C., Seidel, R., Thomann, R., Speck, T., 2012. Comparison of different methods for the detection of silica inclusions in plant tissues, International Journal of Plant Sciences 173, 229-238.

Bonhomme, V., Prasad, S., Gaucherel, C., 2013. Intraspecific variability of pollen morphology as revealed by elliptic Fourier analysis, Plant Systematics and Evolution 299, 811-816.

Cao, D., Jiang, S., Wu, Y., Zhu, S., 2013. Novel invariant Zernicke moments as a shape descriptor for machine vision, in: Lin, X., Zheng, J. (Eds.), 2013 International Conference on Optical Instruments and Technology: Optoelectronic imaging and Processing Technology, Proceedings of SPIE, 8 pgs.

Carlo, J.M., Barbeitos, M.S., Lasker, H.R., 2011. Quantifying complex shapes: Elliptical Fourier analysis of octocoral sclerites, The Biological Bulletin 220, 224-237.

Chavez, S.J., Thompson, R.G., 2006. Early Maize on the Copacabana Peninsula: implications for the archaeology of the Lake Titicaca Basin, in: Staller, J., Tykot, R., Benz, B. (Eds.), Histories of Maize: Multidisciplinary Approaches to the Prehistory, Linguistics, Biogeography, Domestication, and Evolution of Maize, Academic Press, Burlington, Massachusetts, pp. 415-428.

Chitwood, D.H., Kumar, R., Headland, L.R., Ranjan, A., Covington, M.F., Ichihashi, Y., Fulop, D., Jimenez-Gomez, J.M., Peng, J., Maloof, J.N., Sinha, N.R., 2013. A 
quantitative genetic basis for leaf morphology in a set of precisely defined tomato introgression lines, Plant Cell 25, 2465-2481.

Cuthrell, R.Q., 2013. An Eco-Archaeological Study of Late Holocene Indigenous Foodways and Landscape Management Practices at Quiroste Valley Cultural Preserve, San Mateo County, California, Ph.D. dissertation, Dept. of Anthropology, University of California, Berkeley.

Dai, S.J., Huang, H., Gao, Z.Y., Li, K., Xiao, S.M., 2009. Vehicle-logo recognition method based on Tchebichef moment invariants and SVM, 2009 WRI World Congress on Software Engineering, Proceedings 3, 18-21.

Datta, R., Li, J., Wang, J.Z., 2005. Content-based image retrieval: Approaches and trends of the new age, MIR '05 Proceedings of the 7th ACM SIGMM International Workshop on Multimedia Information Retrieval, 253-262.

Dayanandan, P., Kaufman, P.B., Franklin, C.I., 1983. Detection of silica in plants, American Journal of Botany 70, 1079-1084.

del Pozo-Banos, M., Ticay-Rivas, J.R., Alonso, J.B., Travieso, C.M., 2015. Features extraction techniques for pollen grain classification, Neurocomputing 150, 377-391.

Descles, J., Vartanian, M., Harrak, A.E., Quinet, M., Bremond, N., Sapriel, G., Bibette, J., Lopez, P.J., 2008. New tools for labeling silica in living diatoms, New Phytologist $177,822-829$.

Dimitrovski, I., Kocev, D., Loskovska, S., Dzeroski, S., 2011. Hierarchical classification of diatom images using ensembles of predictive clustering trees, Ecological Informatics 7, 19-29.

du Buf, H., Bayer, M.M., 2002. Automatic Diatom Identification, Series in machine Perception and Artificial Intelligence Volume 51, World Scientific, River Edge, New Jersey.

Dujardin, J.-P., Kaba, D., Solano, P., Dupraz, M., McCoy, K.D., Jaramillo-O, N., 2014. Outline-based morphometrics, an overlooked method in arthropod studies? Infection Genetics and Evolution 28, 704-714.

Dutta, I., Banerjee, S., De, M., 2013. Shape descriptors in morphological galaxy classification, International Journal on Advanced Computer Theory and Engineering 2, 136-140.

Essendelft, D.V., 2013. Rapid detection of sub-scale particle features using invariant harmonic wavelet descriptors, Powder technology 235, 747-755.

Evett, R.R., Cuthrell, R.Q., 2013. Phytolith evidence for a grass-dominated prairie landscape at Quiroste Valley on the central coast of California, California Archaeology 5, 319-335. 
Fishkis, O., Ingwersen, J., Lamers, M., Denysenko, D., Streck, T., 2010. Phytolith transport in soil: A field study using fluorescent labeling, Geoderma 157, 27-36.

Fisk, J.D., Batten, R., Jones, G., O'Reilly, J.P., Shaw, A.M., 2005. pH dependence of the crystal violet adsorption isotherm at the silica-water interface, Journal of Physical Chemistry B 109, 14475-14480.

Friedrichs, L., 2013. A simple cleaning and fluorescent staining protocol for recent and fossil diatom frustules, Diatom Research 28, 317-327.

Friedrichs, L., Maier, M., Hamm, C., 2012. A new method for exact three-dimensional reconstructions of diatom frustules, Journal of Microscopy 248, 208-217.

Garcia-Ordas, M.T., Alegre, E., Gonzalez-Castro, V., Garcia-Ordas, D., 2014. aZIBO: A new descriptor based in shape moments and rotational invariant features, 2014 22nd International Conference on Pattern Recognition, 2395-2400.

Godefroy, J.E., Bornert, F., Gros, C.I., Constantinesco, A., 2012. Elliptical Fourier descriptors for contours in three dimensions: A new tool for morphometrical analysis in biology, Comptes Rendus Biologies 335, 205-213.

Green, W.A., Little, S.A., Price, C.A., Wing, S.L., Smith, S.Y., Kotrc, B., Doria, G., 2014. Reading the leaves: A comparison of leaf rank and automated areole measurement for quantifying aspects of leaf venation, Applications in Plant Sciences 2, Article \#1400006.

Gries, C., Soltis, P., 2014. Digitized natural history collections records in traditional research, collaborative research, and big data research, Botany 2014, Boise, Idaho.

Groger, C., Sumper, M., Brunner, E., 2008. Silicon uptake and metabolism of the marine diatom Thalassiosira pseudonana: Solid-state 29Si NMR and fluorescence microscopic studies, Journal of Structural Biology 161, 55-63.

Gui, J., Zhang, Q., Hao, L., Bao, X., 2014. Apple shape classification method based on wavelet moment, Sensors and Transducers 178, 182-187.

Han, J.G., Cao, H., Barb, A., Punyasena, S.W., Jaramillo, C., Shyu, C., 2014. A Neotropical Miocene pollen database employing image-based search and semantic modeling, Applications in Plant Sciences 2, Article \#1400030.

Hart, J.P., Matson, R.G., 2009. The use of multiple discriminant analysis in classifying prehistoric phytolith assemblages recovered from cooking residues, Journal of Archaeological Science 36, 74-83.

Hart, J.P., Matson, R.G., Thompson, R.G., Blake, M., 2011. Teosinte inflorescence phytolith assemblages mirror Zea taxonomy, PLoS ONE 6, e18349. 
Holt, K., Allen, G., Hodgson, R., Marsland, S., Flenley, J., 2011. Progress towards an automated trainable pollen location and classifier system for use in the palynology laboratory, Review of Palaeobotany and Palynology 167, 175-183.

ICPN Working Group, Madella, M., Alexandre, A., Ball, T., 2005. International Code for Phytolith Nomenclature 1.0, Annals of Botany 96, 253-260.

Iwata, H., Ukai, Y., 2002. SHAPE: A computer program package for quantitative evaluation of biological shapes based on elliptic Fourier descriptors, Journal of Heredity 93, 384-385.

Johansen, D.A., 1940. Plant Microtechnique, McGraw-Hill Book Company, Inc., New York and London.

Kannan, C., Sundaram, T., Palvannan, T., 2008. Environmentally stable adsorbent of tetrahedral silica and non-tetrahedral alumina for removal and recovery of malachite green dye from aqueous solution, Journal of Hazardous Materials 157, 137-145.

Kao, T., Chen, S., Chiou, W., Chuang, Y., Kuo-Huang, L., 2008. Various microscopic methods for investigating the venuloid idioblasts of Pteris grevilleana Wall., Taiwania 53, 394-400.

Kassimi, M.A., El Beqqali, O., 2011. 3D model classification and retrieval based on semantic and ontology, IJCSI International Journal of Computer Science Issues 8, Article \#1694-0814.

Kaufman, P.B., Dayanandan, P., Franklin, C.I., Takeoka, Y., 1985. Structure and function of silica bodies in the epidermal system of grass shoots, Annals of Botany 55, 487-507.

Khotanzad, A., Hong, Y.H., 1990. Invariant image recognition by Zernike moments, IEEE Transactions on Pattern Analysis and Machine Intelligence 12, 489-497.

Kloster, M., Kauer, G., Beszteri, B., 2014. SHERPA: an image segmentation and outline feature extraction tool for diatoms and other objects, BMC Bioinformatics 15.

Koutsoukos, I., 2013. Automated Classification of Pollen Grains from Microscope Images using Computer Vision and Semantic Web Technologies, Diploma Thesis, Intelligent Systems Laboratory, School of Electronic and Computer Engineering, Technical University of Crete, Greece.

Krieger, J.D., 2014. A protocol for the creation of useful geometric shape metrics illustrated with a newly derived geometric measure of leaf circularity, Applications in Plant Sciences 2, \#1400009.

Kucki, M., Landwehr, S., Ruhling, H., Maniak, M., Fuhrmann-Lieker, T., 2006. Lightemitting biological photonic crystals - the bioengineering of metamaterials, in: De La 
Rue, R.M., Viktorovitch, P., Lopez, C., Midrio, M. (Eds.), Photonic Crystal Materials and Devices III, Proceedings of SPIE Volume 6182, 9 pgs.

Kuhl, F.P., Giardina, C.R., 1982. Elliptic Fourier features of a closed contour, Computer Graphics and Image Processing 18, 236-258.

Kumar, N., Belhumeur, P.N., Biswas, A., Jacobs, D.W., Kress, W.J., Lopez, I.C., Soares, J.V.B., 2012. Leafsnap: A Computer Vision System for Automatic Plant Species Identification, in: Fitzgibbon, A., Lazebnik, S., Perona, P., Sato, Y., Schmid, C. (Eds.), Computer Vision - ECCV 2012: 12th European Conference on Computer Vision, Florence, Italy, October 7-13, 2012, Proceedings, Part II, Springer Berlin Heidelberg, pp. 502-516.

Kvilekval, K., Fedorov, D., Obara, B., Singh, A., Manjunath, B.S., 2010. Bisque: A platform for bioimage analysis and management, Bioinformatics 26, 544-552.

Lagerstrom, R., Arzhaeva, Y., Bischof, L., Haberle, S., Hopf, F., Lovell, D., 2013. A comparison of classification algorithms within the Classifynder pollen imaging system, in: Sun, C., Bednarz, T., Pham, T.D., Vallotton, P., Wang, D. (Eds.), 2013 International Symposium on Computational Models for Life Sciences, American Institute of Physics, Sydney, Australia, pp. 250-259.

Liang, C., Gao, D., Hubert, M., Yin, X., Gao, C., 2011. A boundary-line method for pattern recognition on real particles, Powder Technology 213, 155-161.

Lootens, P., Van Waes, J., Carlier, L., 2007. Description of the morphology of roots of Chicorium intybus L. partim by means of image analysis: Comparison of elliptic Fourier descriptors and classical parameters, Computers and Electronics in Agriculture 58, 164173.

Lu, H.Y., Zhang, J.P., Wu, N.Q., Liu, K.B., Xu, D., Li, Q., 2009. Phytoliths analysis for the discrimination of foxtail millet (Setaria italica) and common millet (Panicum miliaceum), PLoS ONE, e4448.

Macrini, D.A., 2003. Indexing and Matching for View-Based 3-D Object Recognition Using Shock Graphs, Master's Thesis, Department of Computer Science, University of Toronto, Canada.

Mahi, H., Kaouadji, M., 2014. Shape-texture features for the VHSR satellite images classification using the MLP neural net, EARSeL eProceedings 13, 67-76.

Mander, L., Baker, S.J., Belcher, C.M., Haselhorst, D.S., Rodriguez, J., Thorn, J.L., Tiwari, S., Urrego, D.H., Wesseln, C.J., Punyasena, S.W., 2014. Accuracy and consistency of grass pollen identification by human analysts using electron micrographs of surface ornamentation, Applications in Plant Sciences 2, Article \#1400031. 
Mander, L., Li, M., Mio, W., Fowlkes, C.C., Punyasena, S.W., 2013. Classification of grass pollen through the quantitative analysis of surface ornamentation and texture, Proceedings of the Royal Society B 280, Article \#20131905.

Marcos, J.V., Cristobal, G., 2013. Texture classification using discrete Tchebichef moments, Journal of the Optical Society of America A 30, 1580-1591.

Marcos, J.V., Nava, R., Cristobal, G., Redondo, R., Escalante-Ramirez, B., Bueno, G., Deniz, O., Gonzalez-Porto, A., Pardo, C., Chung, F., Rodriguez, T., 2015. Automated pollen identification using microscopic imaging and texture analysis, Micron 68, 36-46.

Marx, V., 2013. The big challenges of big data, Nature 498, 255-260.

Mebatsion, H.K., Paliwal, J., Jayas, D.S., 2012. Evaluation of variations in the shape of grain types using principal components analysis of the elliptic Fourier descriptors, Computers and Electronics in Agriculture 80, 63-70.

Mebatsion, H.K., Paliwal, J., Jayas, D.S., 2012. A novel, invariant elliptic Fourier coefficient based classification of cereal grains, Biosystems Engineering 111, 422-428.

Milanesi, C., Costantini, L., Firmati, M., Antonucci, F., Faleri, C., Buracchi, A., Cresti, M., 2014. Geometric morphometry and archaeobotany: Characterisation of grape seeds (Vitis vinifera L.) by analysis of form, Open Access Library Journal 1, e634.

Nixon, M.S., Aguado, A.S., 2002. Feature Extraction and Image Processing, Newnes, Oxford, Great Britain.

Oleschko, K., Parrot, J.-F., Ronquillo, G., Shoba, S., Stoops, G., Marcelino, V. 2004. Weathering: Toward a fractal quantifying, Mathematical Geology 36, 607-627.

Orru, M., Grillo, O., Lovicu, G., Venora, G., Bacchetta, G., 2013. Morphological characterisation of Vitis vinifera L. seeds by image analysis and comparison with archaeological remains, Vegetation History and Archaeobotany 22, 231-242.

Out, W.A., Grau, J.F.P., Madella, M., 2014. A new method for morphometric analysis of opal phytoliths from plants, Microscopy and Microanalysis 20, 1876-1887.

Oyama, P., 2014. Method for coffee beans classification through digital images using selected morphological, colour and textural attributes, Master's thesis, Signal Processing and Instrumentation, Escola de Engenharia de Sao Carlos, Brazil.

Piperno, D.M.P.D.R., Dinan, E.H., Umalauf, M., Zhao, Z., Benfer, R.A., 1995. Distinguishing rice (Oryza sativa L.) from wild Oryza species through phytolith analysis: Results of preliminary research, Economic Botany 49, 183-196.

Redondo, R., Bueno, G., Chung, F., Nava, R., Marcos, J.V., Cristobal, G., Rodriguez, T., Gonzalez-Porto, A., Pardo, C., Deniz, O., Escalante-Ramirez, B., 2015. Pollen 
segmentation and feature evaluation for automatic classification in bright-field microscopy, Computers and Electronics in Agriculture 110, 56-69.

Reig-Bolano, R., Marti-Puig, P., Gallego, E., Masferrer, G., Lombarte, A., Ferrer-Arnau, L., Parisi-Baradad, V., 2011. Feature selection for analyzing and retrieving fish otoliths using Elliptic Fourier descriptors of shapes, 2011 7th International Conference on Next Generation Web Services Practices, IEEE, Salamanca, Spain, pp. 290-295.

Russ, J., 2011. The Image Processing Handbook, 6 ed., CRC Press, Taylor and Francis Group, Boca Raton, Florida.

Russ, J.C., Rovner, I., 1989. Stereological identification of opal phytolith populations from wild and cultivated Zea, American Antiquity 54, 784-792.

Sebastian, T.B., Klein, P.N., Kimia, B.B., 2004. Recognition of shapes by editing their shock graphs, IEEE Transactions on Pattern Analysis and Machine Intelligence 26, 550571.

Selvarajah, S., Kodituwakku, S.R., 2011. Performance evaluation of shape analysis techniques, ARPN Journal of Systems and Software 1, 12-18.

Severa, L., Buchar, J., Nedomova, S., 2012. Shape and size variability of roasted arabica coffee beans, International Journal of Food Properties 15, 426-437.

Sheets, H.D., Covino, K.M., Panasiewicz, J.M., Morris, S.R., 2006. Comparison of geometric morphometric outline methods in the discrimination of age-related differences in feather shape, Frontiers in Zoology 3, unpaginated.

Siddiqi, K., Shokoufandeh, A., Dickinson, S.J., Zucker, S.W., 1999. Shock graphs and shape matching, International Journal of Computer Vision 35, 13-32.

Soukup, M., Martinka, M., Cigan, M., Ravaszova, F., Lux, A., 2014. New method for visualization of silica phytoliths in Sorghum bicolor roots by fluorescence microscopy revealed silicate concentration-dependent phytolith formation, Planta 240, 1365-1372.

Suzuki, K., Fujiwara, H., Ohta, T., 2015. The evaluation of macroscopic and microscopic textures of sand grains using elliptic Fourier and principal component analysis:

Implications for the discrimination of sedimentary environments, Sedimentology (TBD).

Tooke, T.R., Coops, N.C., Goodwin, N.R., Voogt, J.A., 2009. Extracting urban vegetation characteristics using spectral mixture analysis and decision tree classifications, Remote Sensing of Environment 113, 398-407.

Tsolakidis, D.G., Kosmopoulos, D.I., Papadourakis, G., 2014. Plant leaf recognition using Zernike moments and histogram of oriented gradients, in: Likas, A., Blekas, K., Kalles, D. (Eds.), Artificial Intelligence: Methods and Applications. 8th Hellenic Conference on AI, SETN 2014, LNCS 8445, Ioannina, Greece, pp. 406-417. 
Vieira, A.R., Neves, A., Sequeira, V., Paiva, R.B., Gordo, L.S., 2014. Otolith shape analysis as a tool for stock discrimination of forkbeard (Phycis phycis) in the Northeast Atlantic, Hydrobiologia 728, 103-110.

Webb, R.H., 1996. Confocal optical microscopy, Reports on Progress in Physics 59, 427-471.

Williams, K., Munkvold, J., Sorrells, M., 2013. Comparison of digital image analysis using elliptic Fourier descriptors and major dimensions to phenotype seed shape in hexaploid wheat (Triticum aestivum L.), Euphytica 190, 99-116.

Williams, S.T., Hall, A., Kuklinski, P., 2012. Unraveling cryptic diversity in the Indo-west Pacific gastropod genus Lunella (Turbinidae) using elliptic Fourier analysis, American Malacological Bulletin 30, 189-206.

Wu, Y., Wang, C.S., 2009. Extended depth of focus image for phytolith analysis. Journal of Archaeological Science 36, 2253-2257.

Yang, M., Kpalma, K., Ronsin, J., 2008. A Survey of Shape Feature Extraction Techniques, in: Yin, P. (Ed.), Pattern Recognition, IN-TECH, pp. 43-90.

Zhang, J., Lu, H., Wu, N., Yang, X., Diao, X., 2011. Phytolith analysis for differentiating between foxtail millet (Setaria italica) and green foxtail (Setaria viridis), PLoS ONE 6, e19726.

Zhao, M., Tang, Z., Liu, P., 2008. Removal of methylene blue from aqueous solution with silica nano-sheets derived from vermiculite, Journal of Hazardous Materials 158, 43-51.

Zhao, Z., Pearsall, D.M., R. A. Benfer, J., Piperno, D.R., 1998. Distinguishing rice (Oryza sativa Poaceae) from wild Oryza species through phytolith analysis, II: Finalized Method, Economic Botany 52, 134-145.

\section{Figure Captions}

Figure 1. Elements of an optimal quantitative phytolith analysis and classification system, with examples of component parameters and relevant research and analytical topics. 
Figure 2. Example of transmitted light microscopy digital image input and computer-processed output from a semi-automated system used to segment objects and to distinguish phytoliths from non-phytolith objects based on color. Phytolith extract from soil was exposed to crystal violet and methylene blue dye, staining non-phytolith amorphous silica a blue color. A composite image produced from a Z-stack (A) was processed to segment objects and replace object color with the average color value of the polygon (B). Using a color threshold, objects stained blue were excluded from the image, leaving polygons representing unstained particles (C). In a later step, particles below a certain size threshold were also removed from the image. (Cuthrell 2013; Evett and Cuthrell 2013).

Figure 3. Workflow sequence of an optimal computer-assisted phytolith analysis and classification system, consisting of a data acquisition and analytical software package integrated with an online international phytolith database. A/P - archaeological or paleoecological phytolith data; Mod - modern phytolith data. 


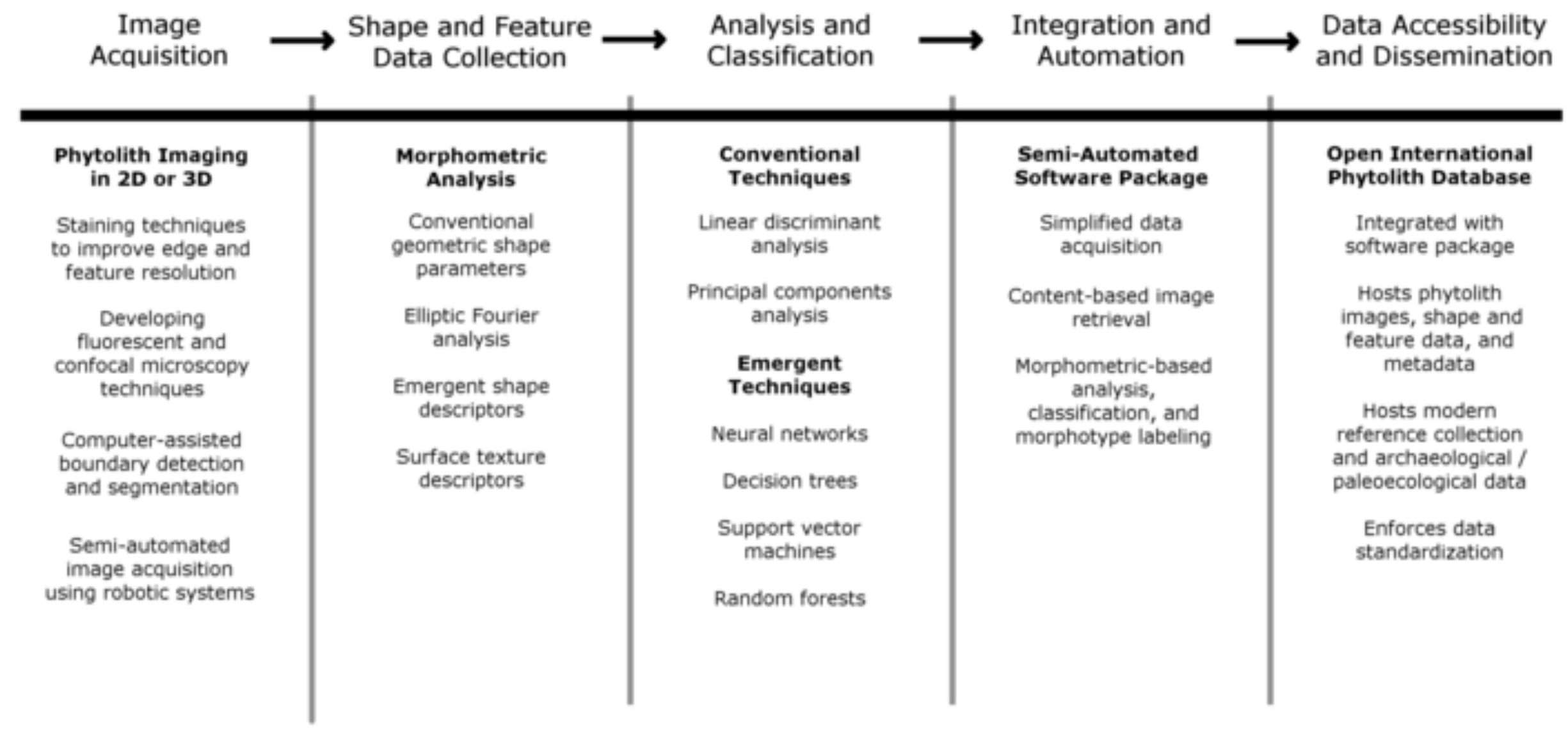




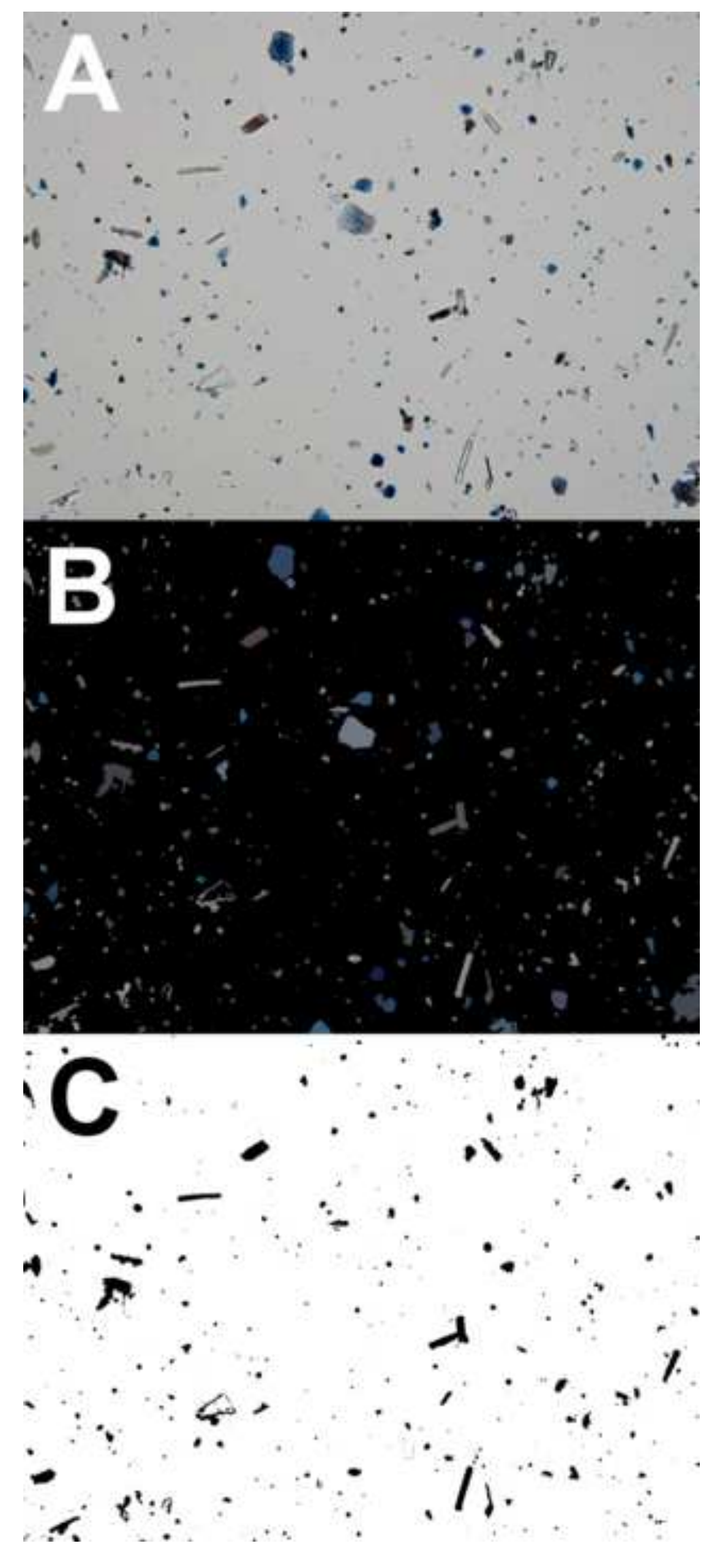

Figure 2 


\section{Data Acquisition} and Analysis

\section{Classification}

\section{Database Integration}

$A / P$, Mod

Content-based image

retrieval

Acquire images, perform morphometric analysis on 2D

images or 3D models containing single or multiple ancient or modern phytoliths

A/P, Mod Automatic assignment of morphotype labels

Morphometric analysis and classification system for anatomical and taxonomic identification Morphometric analysis

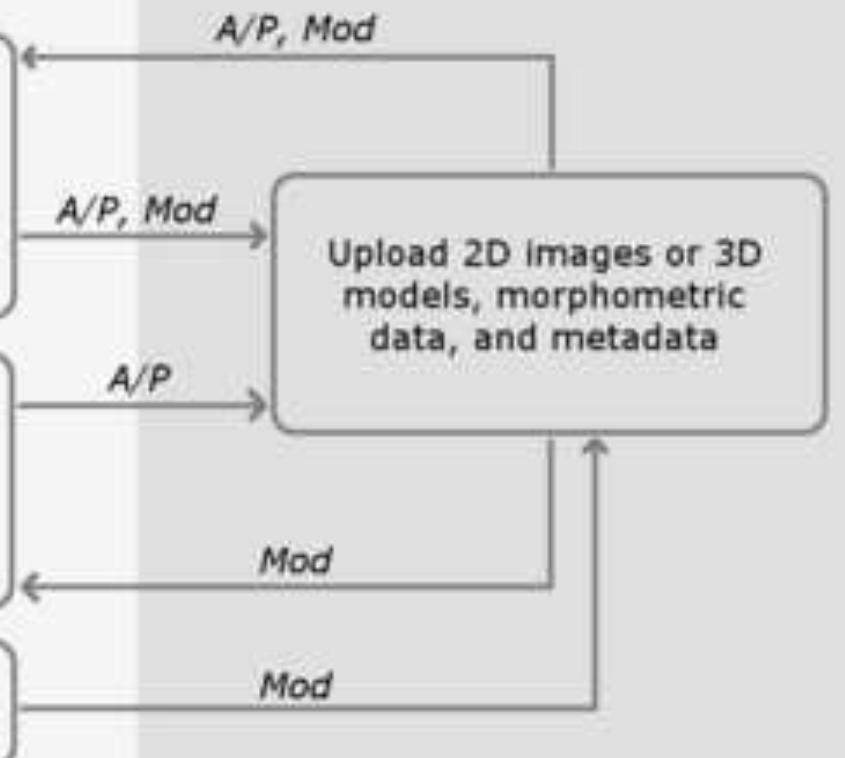

